\title{
Infectious complications in the Southern Tasmanian kidney transplant population
}

\author{
N Abeysekera ${ }^{1}$, A Graver $^{2}$, L Cooley ${ }^{1,3}$, G Kirkland $^{2}$, MD Jose ${ }^{1,2}$ \\ ${ }^{1}$ School of Medicine, University of Tasmania, Australia \\ ${ }^{2}$ Renal Unit, Royal Hobart Hospital, Tasmania, Australia \\ ${ }^{3}$ Infectious Disease Unit, Royal Hobart Hospital, Tasmania, Australia
}

Short Running Title : Infections in Kidney Transplant Population

Word count: 3229

Correspondence to :

Natasha Abeysekera

School of Medicine,

University of Tasmania

Private Bag 96

Hobart TAS 7000

This is the author manuscript accepted for publication and has undergone full peer review but has not been through the copyediting, typesetting, pagination and proofreading process, which may lead to differences between this version and the Version of Record. Please cite this article as doi: $10.1111 /$ nep.13482

This article is protected by copyright. All rights reserved. 


\section{na13@utas.edu.au}

\section{$\underline{\text { Abstract }}$}

Aim: Examine the incidence of suspected and proven infections, the range of infections, antimicrobial use, hospital admissions in kidney transplant recipients in southern Tasmania. Method: An audit of the medical records of kidney transplant recipients managed by the Royal Hobart Hospital for the period $1^{\text {st }}$ of January 2015 to $31^{\text {st }}$ of December 2016. Data was collected on positive microbiological investigations, antimicrobial use and hospital admissions.

Results: Of the 151 evaluable kidney transplant recipients, there were 339 episodes of suspected infection in $95(63 \%)$ patients with a preponderance of urinary tract infections. Overall these 95 kidney transplant recipients received a total of 249 courses of antimicrobials, with predominantly monotherapy $(n=101,65 \%)$. There were eleven vaccine preventable infections, including herpes zoster $(n=7)$, Influenza A $(n=3)$ and invasive pneumococcal disease $(n=1)$. Hospitalisation was required for 50 infectious episodes, for a total of 227 admitted bed days (median 4; IQR 2-7; range 1-18 days).

Conclusion: In conclusion, episodes of infection, hospitalization, antimicrobial use and development of multi-resistant organisms are common following kidney transplantation in this southern Tasmanian cohort. This study has identified several areas of focus for improved patient care including antimicrobial management of urinary tract infections, implementation of programmes to vaccinate KTx prior to transplantation, and development of transplantation specific antimicrobial stewardship programmes.

Key Words: Tasmania, Renal Transplant, Infection, Antibiotic Resistance, Antimicrobial stewardship, Vaccination

Abstract word count: 222 


\section{$\underline{\text { Main Text }}$}

\section{Introduction}

With rising rates of end-stage kidney disease, renal replacement therapy, including dialysis and kidney transplantation, is becoming increasingly prevalent. In 2017, kidneys were the most frequently transplanted organ in Australia with 832 transplants performed ${ }^{1}$. Kidney transplant recipients (KTx) are highly susceptible to infections, of which the most common are urinary tract infections $\mathrm{s}^{2-5}$. Parasuraman et $\mathrm{al}^{6}$ suggest the high risk of infection seen in KTx was due to a complex relationship between the net state of immunosuppression and epidemiological exposure to infection. To that effect, infections in the KTx population may have significant consequences, including graft rejection and death ${ }^{7,8}$.

The 2009 Kidney Disease: Improving Global Outcomes (KDIGO) Clinical Practice Guidelines for the Care of Kidney Transplant Recipients provides evidence-based recommendations for the prevention and management of infections arising in the KTx population ${ }^{9}$. Despite being published nearly a decade ago; infections remain a highly prevalent issue.

With the continued emergence of antimicrobial resistance, it is more important than ever that the management of infections in KTx are consistent with contemporary evidence-based guidelines. To assess our current management of infectious complications in KTx, we evaluated the prevalence and type of infections, hospital admissions and antimicrobial use in the southern Tasmanian renal transplant population.

\section{Methods}

\section{Population}

A retrospective audit of patients receiving care at the Royal Hobart Hospital following renal transplantation (including kidney-pancreas transplantation) was undertaken over a 2-year period, $1^{\text {st }}$ of January 2015 to $31^{\text {st }}$ of December 2016. Details of microbiology testing, test results, antimicrobial use and indication, and hospital admissions were recorded from patient electronic medical records. 
Positive results from active surveillance cultures or testing, including rectal and nasal swabs for carriage of multi-resistant organisms and urine for asymptomatic bacteriuria were recorded but excluded from analysis of infections.

\section{Definitions}

Infections were defined using the Centre for Disease Control and the National Healthcare Safety Network Guidelines ${ }^{10}$. Cytomegalovirus (CMV) disease was defined according to American Society of Transplantation criteria ${ }^{11}$. BK virus associated nephropathy was defined according to the American Society of Transplantation definitions ${ }^{12}$. Primary renal disease categories were assigned based on International Statistical Classification of Diseases - 10 $(\mathrm{ICD}-10)^{13}$. Patients lost to follow up, defined as no attendance to clinic during the study period.

\section{Unit Prophylaxis Regimens}

Prophylaxis of infections follows the KDIGO guidelines ${ }^{9}$. CMV prophylaxis was determined pre-transplant based on recipient CMV-IgG status. All CMV seropositive recipients received 3 months valganciclovir prophylaxis, regardless of donor status, whilst CMV seropositive donor to CMV seronegative recipients receive 6 months valganciclovir prophylaxis. All KTx received at least 6 months of trimethoprim-sulphamethoxazole immediately post-transplant and for at least 6 weeks after treatment for acute rejection to prevent Pneumocystis jirovecii pneumonia. Antimicrobials prescribed for prophylaxis were excluded from the analysis.

\section{Statistical Analysis}

Statistical analysis was conducted using Stata 15 software (StataCorp, USA). Categorical variables were assessed using Chi-squared or Fisher's exact test and were considered significant if $\mathrm{p}<0.05$. This study was approved by the Tasmanian Health and Medical Research Ethics Committee (approval: H0016339) with informed patient consent waived.

\section{Results}

\section{Population}


The southern Tasmanian KTx population comprised 162 living patients on the $1^{\text {st }}$ of January 2015 (Figure 1). Eleven patients were lost to follow up (8\%). Twenty patients (12\%) received their transplant during the study had a follow up range of 4 to 718 days (median 436; IQR 242 - 625). One hundred and thirty-one patients (86.75\%) received their transplant greater than 24 months prior to the study, with a range of 734 to 12418 days (median 2831; IQR 1686 - 4623). Six patients died during the study, one as a result of sepsis.

There were 151 evaluable KTx of which 63 (42\%) were female and had an average age of 52.6 years at transplantation (Table 1). Seventy-one (47\%) received transplants from living donors, 66 (43.7\%) from deceased donors, 10 (6.6\%) received kidney-pancreas transplants and the recipient status was unknown in four $(2.7 \%)$ patients. One hundred and twenty-seven (84\%) patients received their first transplant, 22 (14.6\%) received their second transplant and $2(1.3 \%)$ received their third transplant. The most frequent cause of kidney failure by primary renal disease category was glomerulonephritis $(n=50,38 \%)$ and forty-five patients $(29.8 \%)$ had diabetes. The average human leuckocyte antigen(HLA) mismatches in the KTx group investigated for infections and without was 3.3 and 3 respectively. Most patients $(n=142$, 98\%) received a calcineurin inhibitor, antimetabolite and prednisolone as part of their initial immunosuppression regimen. Two patients (1.4\%) did not receive a calcineurin inhibitor. The most common calcineurin inhibitor used was tacrolimus $(n=131,91 \%)$ and the most common anti-metabolite used was mycophenolate mofetil ( $n=142,98 \%)$. The most common immunosuppression regimen $(\mathrm{n}=65,45 \%)$ provided was tacrolimus, mycophenolate mofetil and prednisolone.

\section{Infectious Episodes}

Of the $151 \mathrm{KTx}, 95$ (62\%) underwent investigations for infection, with a total of 339 episodes investigated, including asymptomatic and symptomatic infections, during the 2-year study period. The rate of suspected infections in the 2-year study period was 2.2 episodes per patient. Of the patients who were investigated for infectious episodes, fifty-eight (61\%) were investigated for more than 1 episode. Patients who received their transplant less than 24 months prior to the study had a rate of 1.9 infectious episodes per patient per year. There 
were 20 infectious episodes investigated in patients who received their transplant less than 12 months prior to the study, which were predominantly asymptomatic bacteriuria $(n=9)$. Females were more likely to be assessed for an infectious episode ( $\mathrm{p}=0.04)$. A total of 124 episodes $(37 \%)$ fulfilled the case definition for infection were confirmed by microbiological testing with the commonest site of infection being urinary tract (UTI) $(n=64)$, followed by respiratory tract $(n=26)$ and skin and soft tissue $(n=15)$.

Of the 82 symptomatic patients assessed for UTI, 69 had a urinary tract pathogen isolated. $E$ coli was the predominate isolate ( $\mathrm{n}=32,46 \%$ of isolates), followed by E faecalis $(\mathrm{n}=17$, $24.6 \%)$ and Klebsiella species $(n=6,8.7 \%)$. These were also the predominate organisms from positive cultures in patients with asymptomatic bacteriuria, with $E$ coli comprising $27 \%$ and E faecalis $23 \%$ of positive cultures, respectively.

Seventy-one patients were assessed for respiratory tract infection (RTI), of which $31 \%$ $(n=24)$ detected an infection, $58 \%(n=41)$ had a negative test and $8 \%(n=6)$ detected a nonpathogenic organism. The majority of infections were viral $(n=14)$, including Rhinovirus ( $\mathrm{n}=6)$ and Influenza A (n=3). Bacterial RTIs comprised Haemophilus $\mathrm{sp} .(\mathrm{n}=3) M$ tuberculosis $(\mathrm{n}=1)$ and $K$. pneumoniae $(\mathrm{n}=1)$.

Twenty-four patients were investigated for skin and soft tissue infections, of whom 19 (41\%) had a pathogen isolated. These included varicella zoster virus ( $\mathrm{n}=7,54 \%)$, Staphylococcus aureus $(\mathrm{n}=5)$ and Pseudomonas aeruginosa $(\mathrm{n}=1)$.

Eleven patients were investigated for gastro-intestinal infection. Nine cases (54\%) had a pathogen detected, including 2 with dual infection. Pathogens included $C$ difficile $(\mathrm{n}=6)$ Campylobacter $\mathrm{sp}(\mathrm{n}=3)$ and Norovirus $(\mathrm{n}=2)$.

Miscellaneous infections included four blood stream infections (S pneumonia, Campylobacter coli, Morganella morganii and one mixed infection due to Bacteroides fragilis and Enterococcus hirae). Five bone and joint infections were identified 
(Staphylococcus aureus [n=1], Coagulase negative staphylococcus [n=3] and Staphylococcus lugdenensis $[\mathrm{n}=1])$.

Nineteen multi-resistant organisms (MROs) were cultured. Thirteen were from screening specimens, including urine and faecal swabs, and six were detected from clinical specimens. MROs included Enterobacteriaceae with extended-spectrum ß-lactamases (n=15), vancomycin-resistant Enterococci $(\mathrm{n}=3)$ and metallo--3-lactamase bla $_{\mathrm{IMP}-1}(\mathrm{n}=1)$. Methicillin resistant Staphylococcus aureus colonisation was not detected. Three patients had CMV viraemia, none of whom fulfilled the criteria for CMV viral syndrome. Four patients had BK viraemia without BK nephropathy.

\section{Antimicrobial prescribing}

Fifty-two patients (34\%) received an antimicrobial agent during the period of the study. In total, 249 antimicrobials were prescribed for $156(46 \%)$ episodes of suspected infection. Monotherapy was used in $65 \%$ of episodes, and three or more antimicrobials were used in $17 \%$.

Antimicrobials were prescribed for 40 of the 71 suspected respiratory tract infections. Augmentin duo forte $(n=15)$, amoxycillin $(n=13)$, ceftriaxone $(n=10)$ and doxycycline $(n=16)$ were the most commonly prescribed antimicrobials. There was no significant difference in prescribing according to isolation of a pathogen, with $42 \%(n=13)$ of untreated patients and $43 \%(n=17)$ of treated patients having a pathogen isolated. Directed therapy with an antimicrobial active against the isolated organism, occurred in only seven episodes.

Amongst patients with suspected skin or soft tissue infection, 22 antimicrobials were prescribed for $62.5 \%(n=15)$ of episodes. Flucloxacillin was the most commonly prescribed antimicrobial $(n=8)$ followed by antivirals $(n=6)$ and amoxycillin/clavulanic acid $(n=6)$. Ten episodes received directed therapy.

An antimicrobial was prescribed in 67 of 178 (38\%) episodes where a urine specimen was collected. An antimicrobial was prescribed in $17 \%(n=16)$ episodes of asymptomatic 
bacteriuria, and $62 \%(n=51)$ of UTIs. A total of 99 antibiotic courses were used, with amoxycillin the most commonly used antibiotic $(n=23)$, followed by cephalexin $(n=17)$, quinolones $(n=15)$ and amoxycillin/clavulanic acid $(n=7)$. Therapy was directed to the pathogen on 38 occasions.

\section{Hospital Admissions}

There were fifty hospital admissions with suspected infection amongst 15 (10\%) patients, utilising 227 bed days. The median number of admission per patient admitted was three (range 1-7) and the median duration of hospital admission was 4.5 days (range 1-18 days). Site of suspected infection included: renal tract $(n=16)$, respiratory tract $(n=12)$, skin and soft tissue $(n=10)$, gastro-intestinal tract $(n=6)$, bloodstream $(n=5)$ and miscellaneous $(n=1)$.

\section{Discussion}

This study highlights the significant morbidity associated with infection in the KTx population. Almost two thirds of our KTx population were assessed for infection at any time over a two year period, with a rate of 2.2 suspected infections per patient. Twenty patients who received their transplant less than 24 months prior to the end of study had an infection rate of 1.9 episodes/year. Studies evaluating KTx during the year post-transplantation have found similar incidence rates of 1.1-1.5 episodes/year ${ }^{14,15}$. Only one third of infectious episodes were microbiologically confirmed. Whilst only $10 \%$ of patients required admission, this group of patients experienced a median of three admissions over the study period.

As has previously been reported, urinary tract infections compromise the majority of infections in our KTx cohort. They were also the commonest reason for admission to hospital and antibiotic prescription. As in the general population ${ }^{16}$ female KTx were more likely to develop a UTI. Management of UTI, particularly recurrent infections in the KTx population is particularly challenging and also critical as they may be associated with graft failure and increased mortality ${ }^{17,18}$. 
Viral infections predominated in KTx presenting with respiratory and skin infections. Fourteen $(58 \%)$ of the microbiologically confirmed respiratory infections were due to a virus, with rhinovirus predominating. Herpes zoster infection occurred in $4 \%$ of the cohort. BK and CMV viraemia were detected in 8 patients without evidence of disease. Viral infections may indicate excessive immunosuppression and research has suggested that instead of augmenting immunosuppressive therapy it should be reduced ${ }^{19,20}$.

There were eleven vaccine preventable diseases (VPDs), including herpes zoster $(n=7)$, Influenza $A(n=3)$ and invasive pneumococcal disease $(n=1)$. This is consistent with a recent review of Australian patients, that included all forms of renal replacement therapy (RRT), including KT, found 79 deaths due to VPDs, with a higher mortality due to VPD in KTx (4.6\%), than other forms of RRT $(2.4 \%)^{21}$. The prevalence is higher in KTx than the general population, for example herpes zoster has a 10 to 100 fold higher incidence in organ transplant recipients ${ }^{22}$, and close contact with the healthcare system during the pretransplantation work-up period provides a window for intervention. While efficacy of influenza vaccine in patients with chronic kidney disease varies from season to season, cohort studies demonstrated $49 \%$ efficacy of shingles vaccine in patients with chronic and end stage kidney disease ${ }^{23}$. Vaccination status was not assessed in our study, however previous studies at our centre found that no patients had complete vaccination records as per the Australian Immunisation Handbook, and only $41.1 \%, 17.8 \%$ and $2.2 \%$ had received an annual influenza vaccination, complete Pneumococcal and herpes zoster vaccination, respectively ${ }^{24}$.

One third of KTx received an antimicrobial during the study period, and monotherapy was generally used. Of concern, the patients who were prescribed antibiotics received an average of 4.8 antimicrobial courses, and with the exception of UTIs, directed therapy was uncommon. Furthermore our study found that a significant proportion of patients with asymptomatic bacteriuria were treated with antimicrobial agents. Whilst the study was not designed to determine appropriateness of therapy, particularly in empiric antimicrobial use, this data indicates that improvements can be made. Detection of multiple episodes of MROs 
and $C$ difficile infection in the KTx population demonstrates that this level of prescribing is not without consequence. Inappropriate or suboptimal antimicrobial use contributes to the development of antimicrobial resistance and medication-related adverse events, and can lead to poorer outcomes for individual patients with infection ${ }^{25}$. An antimicrobial stewardship (AMS) program targeting the solid organ transplant population has been proposed by So et $\mathrm{al}^{26}$, who identified opportunities to support AMS, including increased infectious diseases consultation and the promotion of education regarding microbiology for involved health care professionals. This is supported by Hamandi et $\mathrm{al}^{27}$ who found infectious diseases consultations decrease the rate of repeat hospital admissions and a significant mortality benefit in the solid organ transplant population.

We acknowledge several limitations present within this study. The retrospective nature of the study limited the amount of data able to be collected. Moreover, the data collected from the electronic medical records were from either the hospital or the renal clinic, and did not include infections determined in General Practice. Finally, the small population size limits the ability to extrapolate differences by statistical analysis.

In conclusion, episodes of infection, hospitalization, antimicrobial use and development of multi-resistant organisms are common following kidney transplantation in this southern Tasmanian cohort. This study has identified several areas in the management of infections in KTx for future research to consider, namely with regards to antimicrobial management of urinary tract infections, implementation of programmes to vaccinate KTx prior to transplantation, and development of transplantation specific antimicrobial stewardship programmes.

\section{$\underline{\text { Acknowledgements }}$}

We wish to acknowledge the kidney donors, recipients and staff that support the kidney transplant program in Southern Tasmania,

\section{Conflict of Interest}


All authors declare no commercial conflict of interest.

\section{$\underline{\text { References }}$}

1. DonateLife. 2017 Australian donation and transplantation activity report.

Available at: DonateLife; 2017. 11p. http://www.donatelife.gov.au/annual-reports, accessed $21^{\text {st }}$ March 2018.

2. Shamila K, Blumberg E. Common Infections in Kidney Transplant Recipients. Clin J Am Soc Nephrol. 2012 Dec;7(12):2058-70.

3. Alangaden GJ, Thyagarajan R, Gruber SA, et al. Infectious complications after kidney transplantation: current epidemiology and associated risk factors. Clin Transplant. 2006 Jul;20(4):401-9.

4. Pellé G, Vimont S, Levy PP, et al. Acute pyelonephritis represents a risk factor impairing long-term kidney graft function. Am J Transplant. 2007 Apr;7(4):899907.

5. Lee JR, Bang H, Dadhania D, et al. Independent risk factors for urinary tract infection and for subsequent bacteremia or acute cellular rejection: a single-center report of 1166 kidney allograft recipients. Transplantation. 2013 Oct;96(8):732-8.

6. Parasuraman R, Samparapungavan D, Venkat KK. Updated principles and clinical caveats in the management of infection in renal transplant recipients. Transplant Rev. 2010 Apr;24(2):43-51.

7. Prakash J, Ghosh B, Singh S, Soni A, Rathore SS. Causes of death in renal transplant recipients with functioning allograft. Indian J Nephrol. 2012 JulAug;22(4):264-268.

8. Ko KS, Cho DO, Ahn JH, et al. Infections after renal transplantation. Transplant Proc. 1994 Aug;26(4):2072-4.

9. Kidney Disease: Improving Global Outcomes(KDIGO) Transplant Work Group. KDIGO clinical practice guideline for the care of kidney transplant recipients. Am J Transplant. 2009 Nov; 9suppl3:S1-155. 
10. Centre for Disease Control and Prevention, National Healthcare Safety Network [Internet]. CDC/NHSN Surveillance Definitions for Specific Types of Infections; 2018 Jan [cited 2018 Jul 1]. Available from :

https://www.cdc.gov/nhsn/pdfs/pscmanual/17pscnosinfdef_current.pdf.

11. Humar A, Michaels M. American Society of Transplantation recommendations for screening, monitoring and reporting of infectious complications in immunosuppression trials in recipients of organ transplantation. Am J Transplant. 2006;6:262-274.

12. Hirsch HH, Randhawa P. BK polyomavirus in solid organ transplantation. Am J Transplant. 2013 Mar; 13 Suppl 4:179-88.

13. World Health Organization [Internet]. International Classification of Diseases; 2016. Diseases of the genitourinary system; 2016 [cited 2018 Jul 1]. Available from: http://www.who.int/classifications/icd/en/.

14. Sousa SR, Galante NZ, Barbosa DA, Pestana JO. Incidence of infectious complications and their risk factors in the first year after renal transplantation. $\mathrm{J}$ Bras Nefrol. 2010 Mar; 32(1):75-82.

15. Maraha B, Bonten H, Van Hooff H, Fiolet H, Buiting AG, Stobberingh EE. Infectious complications and antibiotic use in renal transplant recipients during a 1-year follow-up. Clin Microbiol Infect. 2001 Nov; 7(11):619-25.

16. Al-Badr A, Al-Shaikh G. Recurrent Urinary Tract Infections Management in Women. Sultan Qaboos Univ Med J. 2013 Aug;13(3):359-367.

17. Giessing M. Urinary tract infection in renal transplantation. Arab J Urol. 2012 Jun;10(2):162-168.

18. Nagarajan M, Ramanathan S, Dhanapriya J, Dineshkumar T, Subramaniyan TB, Gopalakrishnan N. Impact of acute kidney injury on renal allograft survival. Ren Fail. 2017 Nov;39(1):40-44.

19. Bohl DL, Koch MJ, Brennan DC. Viral Infections in Renal Transplantation: A Clue to Excessive Immunosuppression. J Bras Nefrol. 2007 Sep;29(3):185-190.

20. Bohl DL, Brennan DC. BK Virus Nephropathy and Kidney Transplantation. Clin J Am Soc Nephrol. 2007 Jul; 2(1):S36-46. 
21. Graver A, Kuo S, Cooley L, Abeysekera N, Kirkland G, Jose MD. What are the infections that Australians on renal replacement therapy die from. Nephrology; 22(3): 44.

22. Pergam SA, Forsberg CW, Boeckh MJ, Maynard C, Limaye AP, Wald A, Smith NL, Young BA. Herpes zoster incidence in a multi-center cohort of solid organ transplant recipients. Transpl Infect Dis. 2012 Feb; 13(1): 15-23.

23. Tseng HF, Luo Y, Shi J, Sy LS, Tartof SY, Sim JJ, Hechter RC, Jacobsen SJ. Effectiveness of herpes zoster vaccine in patients 60 years and older with endstage renal disease. Clin Infect Dis. 2016; 62:462-467.

24. Graver A, Cooley L, Kuo S, Kirkland G, Jose MD. Vaccine preventable disease in immunosuppressed people with kidney disease. Nephrology; 22(3): 43.

25. Australian Commission on Safety and Quality in Health Care. Antimicrobial Stewardship in Australian Health Care 2018. Sydney: ACSQHC; 2018.

26. So M, Yang DY, Bell C, Humar A, Morris A, Husain S. Solid organ transplant patients: are there opportunities for antimicrobial stewardship. Clin Transplant. 2016 Jun; 30(6): 659-68.

27. Hamandi B, Husain S, Humar A, Papadimitropoulos EA. Impact of infectious disease consultation on the clinical and economic outcomes of solid organ transplant recipients admitted for infectious complications. Clin Infect Dis. 2014 Oct 15; 59(8): 1074-82. 


\section{University Library}

\section{- M M I N E R VA A gateway to Melbourne's research publications}

Minerva Access is the Institutional Repository of The University of Melbourne

\section{Author/s:}

Abeysekera, N;Graver, A;Cooley, L;Kirkland, G;Jose, MD

Title:

Infectious complications in the Southern Tasmanian kidney transplant population

\section{Date:}

2019-08-01

Citation:

Abeysekera, N., Graver, A., Cooley, L., Kirkland, G. \& Jose, M. D. (2019). Infectious complications in the Southern Tasmanian kidney transplant population. NEPHROLOGY, 24 (8), pp.849-854. https://doi.org/10.1111/nep.13482.

Persistent Link:

http://hdl.handle.net/11343/285783 